\title{
Heat shock cognate 71 (HSC71) regulates cellular antiviral response by impairing formation of VISA aggregates
}

\author{
Zhigang Liu ${ }^{1,2}$, Shu-Wen $W_{u}{ }^{1}$, Cao-Qi Lei ${ }^{1}$, Qian Zhou ${ }^{1}$, Shu Li ${ }^{1}$, Hong-Bing Shu ${ }^{1}$, Yan-Yi Wang ${ }^{1,3 \bowtie ~}$ \\ ${ }^{1}$ College of Life Sciences, Wuhan University, Wuhan 430072, China \\ ${ }^{2}$ Key Laboratory of Fermentation Engineering (Ministry of Education), Hubei University of Technology, Wuhan 430068, China \\ ${ }^{3}$ State Key Laboratory of Virology, Wuhan Institute of Virology, Chinese Academy of Sciences, Wuhan 430072, China \\ $\triangle$ Correspondence: wangyy@wh.iov.cn
}

Received April 1, 2013 Accepted April 15, 2013

\begin{abstract}
In response to viral infection, RIG-I-like RNA helicases detect viral RNA and signal through the mitochondrial adapter protein VISA. VISA activation leads to rapid activation of transcription factors IRF3 and NF-KB, which collaborate to induce transcription of type I interferon (IFN) genes and cellular antiviral response. It has been demonstrated that VISA is activated by forming prionlike aggregates. However, how this process is regulated remains unknown. Here we show that overexpression of HSC71 resulted in potent inhibition of virus-triggered transcription of IFNB1 gene and cellular antiviral response. Consistently, knockdown of HSC71 had opposite effects. HSC71 interacted with VISA, and negatively regulated virus-triggered VISA aggregation. These findings suggest that HSC71 functions as a check against VISA-mediated antiviral response.
\end{abstract}

KEYWORDS HSC71, VISA, Cellular antiviral response, prion-like aggregate

\section{INTRODUCTION}

Viral infection triggers the innate immune response by inducing type I interferons (IFNs) and other downstream antiviral proteins. However, an overzealous immune response is deleterious to the host when left unchecked (Allen et al., 2011). Conserved viral structures called pathogen-associated molecular patterns (PAMPs) are sensed by several classes of pattern recognition receptors (PRRs), including RIG-I-like receptors (RLRs) and Toll-like receptors (TLRs) (Akira et al., 2006). The
RLRs, including RIG-I and MDA5, are expressed in most cell types and play important roles in recognizing viral RNAs (Andrejeva et al., 2004; Yoneyama et al., 2004). After binding to viral RNA, the N-terminal CARD domains of RIG-I and MDA5 interact with the CARD domain of the mitochondrial adaptor protein VISA (also known as MAVS, IPS-1, and CARDIF), resulting in the activation of transcription factors IRF3 and NF-KB, and subsequent induction of type I IFN genes (Kawai et al., 2005; Meylan et al., 2005; Seth et al., 2005; Xu et al., 2005).

The mechanism by which VISA is activated following viral infection has been recently revealed (Hou et al., 2011). It has been suggested that the binding of viral RNA to the $C$ terminus of RIG-I induces a conformational change that exposes its $\mathrm{N}$-terminal CARD domains for binding to unanchored K63 polyubiquitin chains, leading to activation of RIG-I (Zeng et al., 2010). The activated RIG-I binds to VISA, which catalyzes the conversion of VISA on the mitochondrial membrane to prionlike aggregates, leading to activation of IRF3 in the cytosol (Hou et al., 2011). Various molecules, including Atg5Atg12, NLRX1, gC1qR, PSMA7, Mitofusin 2, AIP4 and RNF5 have been shown to regulate VISA-mediated signaling (Jounai et al., 2007; Moore et al., 2008; Jia et al., 2009; Xu et al., 2009; Yasukawa et al., 2009; You et al., 2009; Zhong et al., 2009). However, whether these molecules regulate VISA aggregation and the in vivo relevance of these molecules to VISA-mediated signaling are unknown.

Heat shock proteins (HSPs) are evolutionarily conserved proteins that are widely expressed in microorganisms and mammalian cells. HSPs are induced by temperature shock as well as other stress signals including viral infection. By serving as intracellular chaperones or acting as regulators of the 
apoptotic machineries, HSPs prevent cell death under stress conditions or upon other physiological and pathological stimuli (Chen and Cao, 2010). Some members of HSPs have been implicated in adaptive and innate immunity (Tsan and Gao, 2004). HSPs of the cytosol such as Hsp70 and Hsp90 have been demonstrated to regulate antigen-processing in adaptive immunity (Srivastava et al., 1998; Li et al., 2002), and the extracellular HSP70 and Hsp90 bind with LPS and potentiate TLR4-mediated inflammatory responses (Triantafilou and Triantafilou, 2004).

In this study, we found that intracellular heat shock cognate 71 (HSC71) prevents VISA to form functional aggregates following viral infection, leading to inhibition of virus-induced IFN- $\beta$ expression and cellular antiviral response. These findings provide insights into the mechanism on how VISA is negatively regulated in the virus-triggered signaling pathways.

\section{RESULTS}

Identification of HSC71 as a negative regulator of virus-triggered signaling

Virus-triggered IFN induction is delicately regulated to avoid excessive immune response. To identify new molecules that negatively regulate the virus-triggered type I IFN expression, we screened $\sim 10,000$ independent human cDNA expression plasmids for their abilities to regulate SeV-induced IFN- $\beta$ promoter activation by reporter assays. These efforts led to the identification of HSC71 (also known as HSPA8), a member of the HSP70 family, which markedly inhibited SeV-triggered activation of the IFN- $\beta$ promoter in a dose-dependent manner (Fig. 1A). We further examined whether other HSP family proteins had similar functions. Among the 12 tested HSP proteins, HSC71 had the most dramatic inhibitory effect on SeVtriggered activation of the IFN- $\beta$ promoter (Fig. S1).

It has been demonstrated that induction of type I IFNs requires coordinated and cooperative action of transcription factors IRF3 and NF-KB (Akira et al., 2006). In reporter assays, overexpression of HSC71 inhibited SeV-triggered activation of ISRE, a conserved enhancer motif recognized by activated IRF3 (Fig. 1B). Consistently, HSC71 inhibited SeV-induced IRF3 dimerization in a dose-dependent manner in 293 cells (Fig. 1B). In reporter assays, overexpression of HSC71 also inhibited SeV-triggered activation of NF-kB (Fig. 1C). In similar experiments, overexpression of HSC71 had no inhibitory role on IFN- $\gamma$-induced activation of the IRF1 promoter (Fig. 1D). Real-time PCR experiments also indicated that overexpression of HSC71 markedly inhibited SeV-induced transcription of endogenous IFNB1, Rantes and TNFa genes (Fig. 1E). Taken together, these data suggest that HSC71 negatively regulates virus-triggered signaling.

\section{Knockdown of HSC71 potentiates virus-induced signaling}

To determine whether HSC71 is involved in regulation of virustriggered type I IFN induction under physiological conditions, we examined the effects of knockdown of HSC71 on virustriggered signaling. We constructed three RNAi plasmids for HSC71 targeting different sites of human HSC71 mRNA (Fig. 2A). Reporter assays indicated that knockdown of HSC71 potentiated SeV-induced activation of the IFN- $\beta$ promoter (Fig. 2B). The potentiation of HSC71 RNAi plasmids on SeVinduced IFN- $\beta$ induction was correlated with their abilities to down-regulate HSC71 expression (Fig. 2A and 2B). Knockdown of HSC71 by RNAi also potentiated SeV-induced ISRE and NF-KB activation (Fig. 2C and 2D). We selected the HSC71-RNAi-\#1 plasmid for additional experiments described below. Knockdown of HSC71 also enchanced virus-triggered IRF3 dimerization (Fig. 2C), which is a hallmark of IRF3 activation. An RNAi off-target HSC71 mutant with three-nucleotide nonsense mutations in the target sequence of the HSC71RNAi-\#1 plasmid, rescued the HSC71-RNAi-\#1-mediated potentiation of SeV-induced activation of the IFN- $\beta$ promoter (Fig. 2E), further confirming that HSC71 plays an important role in SeV-induced IFN- $\beta$ induction. Quantitative real-time PCR experiments confirmed that knockdown of HSC71 could potentiate virus-induced transcription of endogenous IFNB1, Rantes and TNFa genes in 293 cells (Fig. 2F). These data suggest that endogenous HSC71 negatively regulates SeVinduced signaling.

\section{HSC71 negatively regulates cellular antiviral response}

Since HSC71 negatively regulates virus-triggered IFN- $\beta$ induction, we further examined its role in cellular antiviral response. We evaluate the effect of HSC71 on viral replication by plaque assays. We found that overexpression of HSC71 markedly enhanced VSV replication in 293 cells in a dose-dependent manner (Fig. 3A), whereas knockdown of HSC71 had an opposite effect (Fig. 3B). These data suggest that HSC71 negatively regulates cellular antiviral response.

\section{HSC71 regulates virus-induced signaling at the VISA level}

Various components are involved in virus-induced signaling. To determine at which levels that HSC71 regulates virus-induced signaling, we performed reporter assays. As shown in Fig. 4A and 4B, overexpression of HSC71 inhibited VISA- and TBK1mediated but not IRF3-5D- or TRIF-mediated activation of ISRE (Fig. 4A). Knockdown of HSC71 potentiated VISA- but not TBK1- and IRF3-5D-mediated activation of ISRE (Fig. 4B). These results suggest that $\mathrm{HSC71}$ regulates virus-triggered IFN- $\beta$ induction at the level of VISA.

\section{HSC71 impairs recruitment of TRAF2/3/6 to VISA}

Because HSC71 regulates virus-induced signaling at the VISA level, we hypothesized that VISA is the target of HSC71. Endogenous coimmunoprecipitation experiments indicated that HSC71 constitutively interacted with VISA in the absence or presence of SeV infection (Fig. 5A). Confocal microscopy indicated that HSC71 colocolized with VISA at the mitochondria 
A

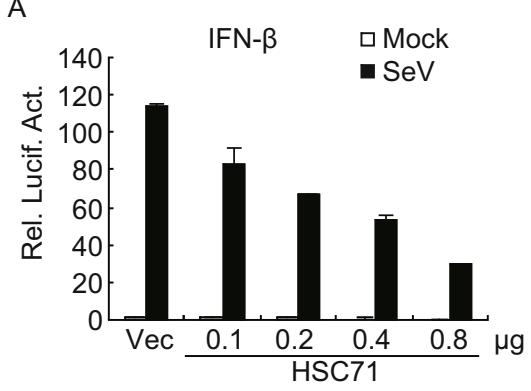

B

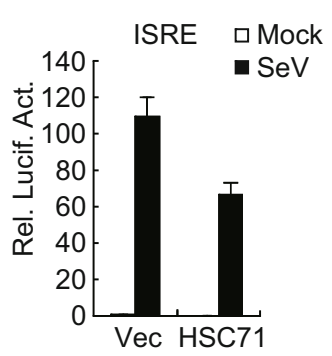

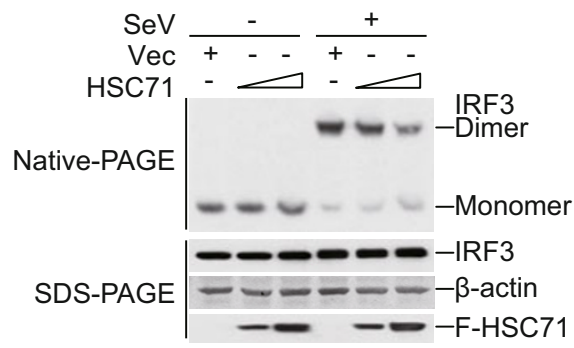

C

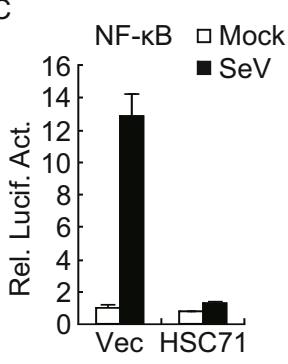

D

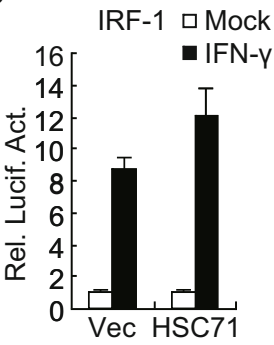

E

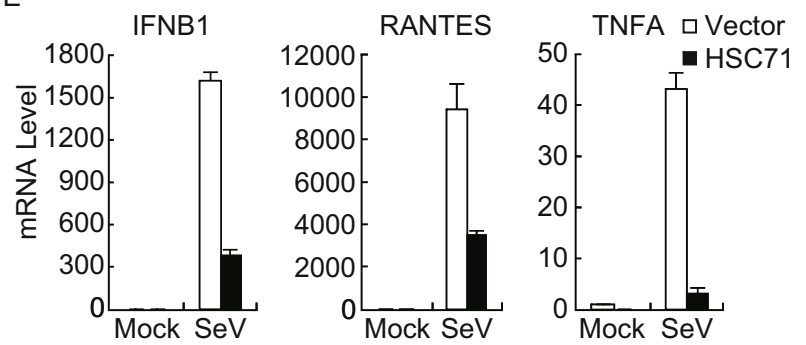

Figure 1. HSC71 inhibits virus-induced signaling. (A) HSC71 inhibits SeV-induced activation of the IFN- $\beta$ promoter. The 293 cells $\left(1 \times 10^{5}\right)$ were transfected with an IFN- $\beta$ promoter reporter $(0.1 \mu \mathrm{g})$, an empty vector or increased amounts of HSC71 expression plasmid. Twenty hours after transfection, cells were left uninfected or infected with SeV for $10 \mathrm{~h}$ before luciferase assays were performed. (B) HSC71 inhibits SeV-induced IRF3 activation. Reporter assays (histograph) were performed similarly as in (A) except that ISRE reporter were used. For the IRF3 dimerization experiments (immunoblots), the 293 cells $\left(2 \times 10^{5}\right)$ were transfected with the indicated plasmids for $24 \mathrm{~h}$. The cells were then left uninfected or infected with SeV for $8 \mathrm{~h}$. Cell lysates were fractionated by native or SDS PAGE and analyzed by immunoblots with the indicated antibodies. (C) HSC71 inhibits SeV-induced NF-KB activation. Reporter assays were performed similarly as in (A) except that NF-KB reporter was used. (D) HSC71 does not inhibit IFN-y-induced activation of the IRF-1 promoter. Experiments were performed similarly as in (A) except the IRF-1 promoter reporter was transfected and IFN- $\mathrm{Y}(100 \mathrm{ng} / \mathrm{mL}) \mathrm{was}$ added into culture medium for $12 \mathrm{~h}$ before luciferase assays were performed. (E) HSC71 inhibits SeV-induced transcription of endogenous IFNB1, RANTES and TNF $\alpha$ genes. The 293 cells $\left(2 \times 10^{5}\right)$ were transfected with an empty vector or HSC71 expression plasmid $(1 \mu \mathrm{g})$. Twenty hours after transfection, cells were left untreated or stimulated with $\mathrm{SeV}$ for $10 \mathrm{~h}$ before quantitative RT-PCR was performed.

before and after SeV infection (Fig. S2). Domain-mapping experiments indicated that both the $\mathrm{N}$ terminus (Nucleotidebinding domain, aa 1-381) and the $C$ terminus (aa 382-464) of HSC71 interacted with VISA, whereas both the CARD domain (aa 1-180) and the transmembrane (TM) domain (aa 509-540) of VISA could interact with HSC71 (Fig. 5B).

After binding with RIG-I, VISA recruits TBK1 indirectly by adapter molecules such as TRAF3, NEMO and TANK or IFIT3 (Tang and Wang, 2010; Liu et al., 2011). We wondered whether HSC71 inhibits virus-triggered signaling through disrupting the interaction of VISA with either its upstream protein RIG-I or downstream kinase TBK1. To test this, we performed competitive coimmunoprecipitation experiments. The results indicated that HSC71 impaired the recruitment of TBK1 to VISA in a dose-dependent manner but not the interaction between VISA and RIG-I (Fig. 5C). Because HSC71 did not down-regulate TBK1 (Fig. 5C) or directly interact with TBK1 (Fig. S3), excluding the possibility that HSC71 targets TBK1 directly to impair the recruitment.

It has been demonstrated that RIG-I and VISA are modified by K63-linked polyubiquitin chains after virus infection (Zeng et al., 2010), and HSC71 interacts with an E3 ubiquitin ligase called CHIP (STUB1) by its C-terminal region (Wang et al., 2012). We determined whether HSC71 regulates polyubiquitination of VISA and RIG-I. As shown in Fig. 5D, overexpression of HSC71 did not regulate polyubiquitination and expression levels of VISA and RIG-I.

In response to viral infection, VISA recruits TRAF2/3/6 directly to trigger the downstream signaling (Nakhaei et al., 2009; Tang and Wang, 2010; Hou et al., 2011). We wondered whether HSC71 inhibits VISA downstream signaling through impairing the recruitment of TRAF2/3/6 to VISA. To test this, we performed the endogenous coimmunoprecipitation experiments with the crude mitochondria isolated from 293 cells in which HSC71 is overexpressed or down-regulated by RNAi knockdown.. The results indicated that overexpression of HSC71 inhibited SeV-induced recruitment of TRAF2/3/6 to VISA, whereas knockdown of HSC71 enhanced the recruitment (Fig. 5E).

\section{HSC71 inhibits formation of VISA aggregates}

Our above coimmunoprecipitation experiments indicated that 
A

HSC71-RNA

Con \#1 \#2 \#3

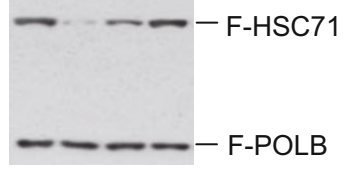

HSC71-RNA

Con \#1 \#2 \#3

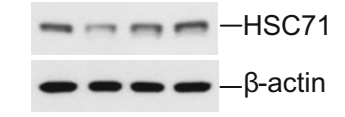

C

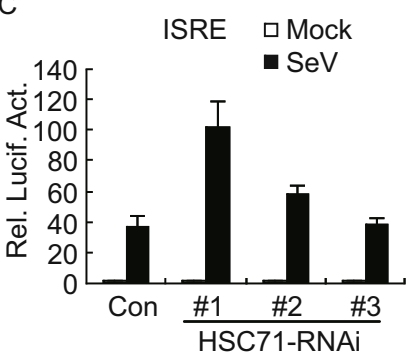

D

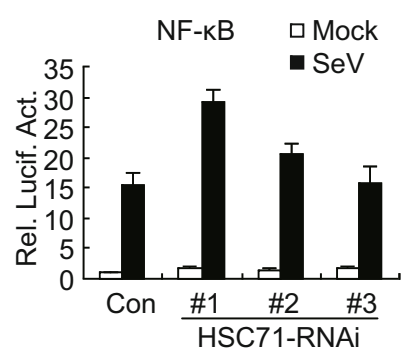

B
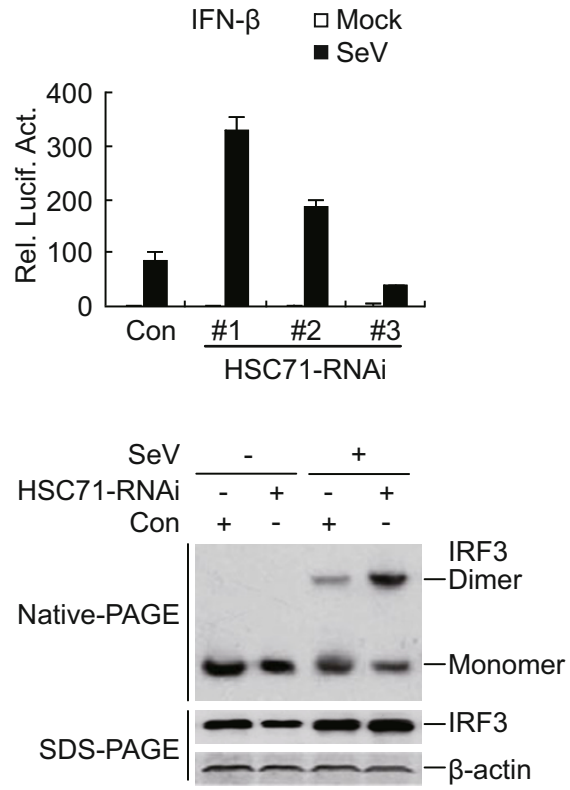

E

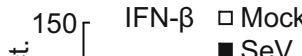

远120

엉 90

כ 60

வ் 30

30

0 -

HSC71-RNAi:
HSC71-WT:

HSC71-Mut:

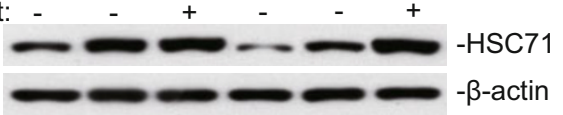

RANTES

TNFa $\square$ Con

F

IFNB1

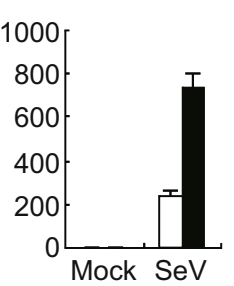

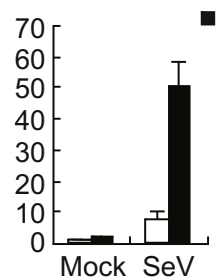

Figure 2. Effects of HSC71 knockdown on virus-induced signaling. (A) Effects of HSC71-RNAi plasmids on expression of HSC71. In the upper panels, the 293 cells $\left(2 \times 10^{5}\right)$ were transfected with the indicated expression $(0.1 \mu \mathrm{g}$ each) and GFP-RNAi control or HSC71RNAi plasmids ( $1 \mu \mathrm{g}$ each) for $36 \mathrm{~h}$ before immunoblot analysis was performed with anti-Flag. In the lower two panels, the 293 cells $\left(2 \times 10^{5}\right)$ were transfected with the indicated RNAi plasmids $(1 \mu \mathrm{g}$ each) for $48 \mathrm{~h}$ before immunoblot analysis was performed with the indicated antibodies. (B) Effects of HSC71-RNAi on SeV-induced activation of the IFN- $\beta$ promoter. The 293 cells $\left(1 \times 10^{5}\right)$ were transfected with the indicated RNAi plasmids $(0.5 \mu \mathrm{g}$ each) for $24 \mathrm{~h}$, and then infected with SeV or left uninfected for $10 \mathrm{~h}$ before reporter assays were performed. (C) Effects of HSC71-RNAi on SeV-induced ISRE activation. Reporter assays (histograph) were performed similarly as in (B) except that ISRE reporter plasmid wes used. For the IRF3 dimerization experiments (immunoblots), Experiments were performed similarly as in (Fig. 1B) except that cells were transfected with HSC71-RNAi or GFP-RNAi control plasmid (1 $\mu \mathrm{g}$ each) for $48 \mathrm{~h}$ before virus infection.(D) Effects of HSC71-RNAi on SeV-induced NF-KB activation. The experiments were similarly performed as in (B) except that NF-KB reporter plasmid were used. (E) Rescue of HSC71-RNAi effect by an RNAi-resistant mutant. The 293 cells $\left(2 \times 10^{5}\right)$ were transfected with HSC71-RNAi plasmid, together with wild-type or RNAi-resistant mutant HSC71 plasmid for $24 \mathrm{~h}$. Cells were then left uninfected or infected with $\mathrm{SeV}$ for $10 \mathrm{~h}$ before luciferase assays. (F) Effects of HSC71-RNAi on SeV-induced transcription of endogenous IFNB1, RANTES and TNF $\alpha$ genes. The 293 cells $\left(2 \times 10^{5}\right)$ were transfected with the indicated RNAi plasmids $(1 \mu \mathrm{g})$. Thirty-six hours after transfection, cells were left uninfected or infected with SeV for $10 \mathrm{~h}$ before RT-PCR experiments were performed. 
A

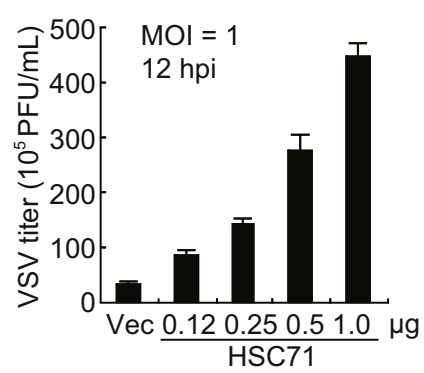

B

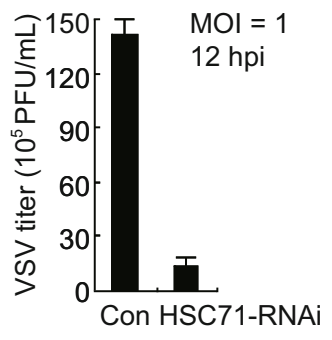

Figure 3. Roles of HSC71 in cellular antiviral response. (A) Overexpression of HSC71 increases VSV replication. The 293 cells $\left(1 \times 10^{5}\right)$ were transfected with an empty vector or increased amounts of HSC71 expression plasmid. Eighteen hours later, cells were further transfected with poly(I:C) $(1 \mu \mathrm{g})$. Twenty-four hours after transfection, cells were infected with VSV [multiplicity of infection (moi), 1], and the supernatants were harvested at $12 \mathrm{~h}$ postinfection. Supernatants were analyzed for VSV production with standard plaque assays. (B) Knockdown of HSC71 inhibits VSV replication. Plaque assays were performed as in (A) except that a control or HSC71-RNAi plasmid $(1 \mu \mathrm{g})$ was transfected.

HSC71 could impair the recruitment of downstream signaling components to VISA following viral infection. Previous studies have demonstrated that prion-like aggregation of VISA on mitochondrial membrane is essential for recruiting downstream proteins and activating IRF3 following viral infection (Hou et al., 2011). To investigate whether HSC71 impairs the recruitment of downstream components to VISA by abrogating virusinduced formation of VISA aggregates, we examined the effects of overexpression or knockdown of HSC71 on formation of VISA aggregates following viral infection. The 293 cells were transfected with HSC71 expression or RNAi plasmid, then infected with $\mathrm{SeV}$ or left uninfected for various times. We then isolated crude mitochondria from these cells by differential centrifugation. The crude mitochondria were resuspended in a sample buffer and then fractionated on semidenaturing detergent agarose gel electrophoresis (SDD-AGE) for detection of

prion-like VISA aggregates by immunoblots (Hou et al., 2011). The results indicated that overexpression of HSC71 reduced SeV-induced formation of VISA aggregates (Fig. 6A), whereas knockdown of HSC71 increased formation of VISA aggregates in response to $\mathrm{SeV}$ infection (Fig. 6B). In these experiments, overexpression or knockdown of HSC71 did not markedly affect the expression levels of VISA before and after SeV infection (Fig. 6A and 6B).

We also performed immunofluorescent microscopy to examine the effect of HSC71 on formation of VISA aggregates. As shown in Fig. S2, HSC71 was mostly colocalized with VISA at mitochondria in both uninfected and SeV-infected cells. In these experiments, SeV infection induced aggregation of VISA, which was partially inhibited by overexpression of HSC71 (Fig. 6C). These results suggest that HSC71 impairs virustriggered formation of VISA aggregates.
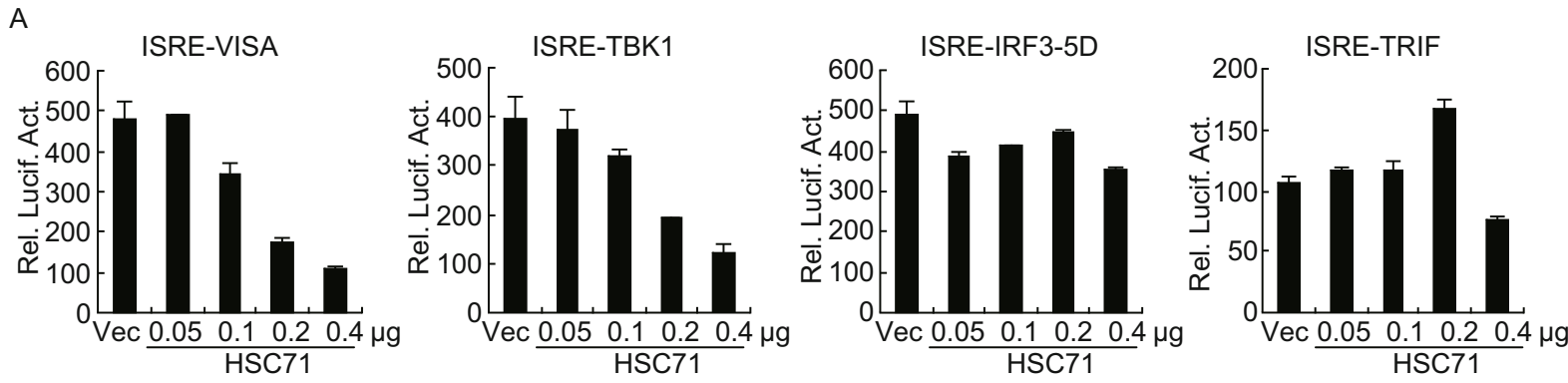

B
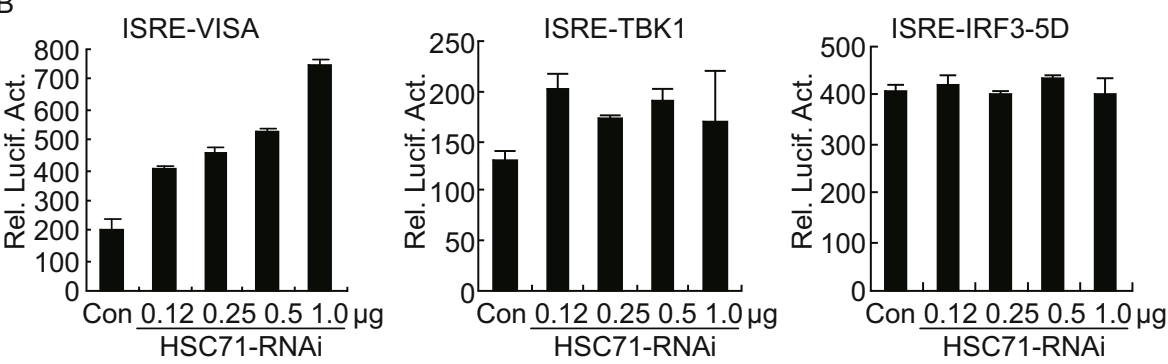

Figure 4. HSC71 regulates virus-induced signaling at the VISA Level. (A) Overexpression of HSC71 inhibits VISA- and TBK1-mediated but not IRF3-5D- and TRIF-mediated activation of ISRE. The 293 cells $\left(1 \times 10^{5}\right)$ were transfected with an ISRE reporter $(0.1 \mu \mathrm{g})$, increased amounts of HSC71 expression plasmid and the indicated expression plasmids $(0.1 \mu \mathrm{g}$ each) for $30 \mathrm{~h}$ before luciferase assays were performed. (B) Knockdown of HSC71 potentiates VISA- but not TBK1- and IRF3-5D-mediated activation of ISRE. Experiments were performed as in (A) except that a control or HSC71-RNAi plasmid $(1 \mu \mathrm{g})$ was transfected. 
Sev: $\frac{-}{\text { IP Ab: } \lg \text { aVISA }} \frac{+}{\lg \text { aVISA }}$

IB: $\alpha \mathrm{HSC71}$

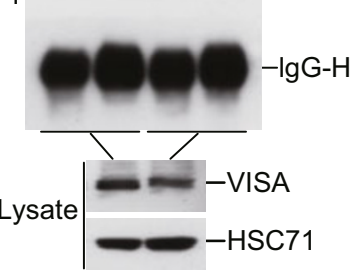

C

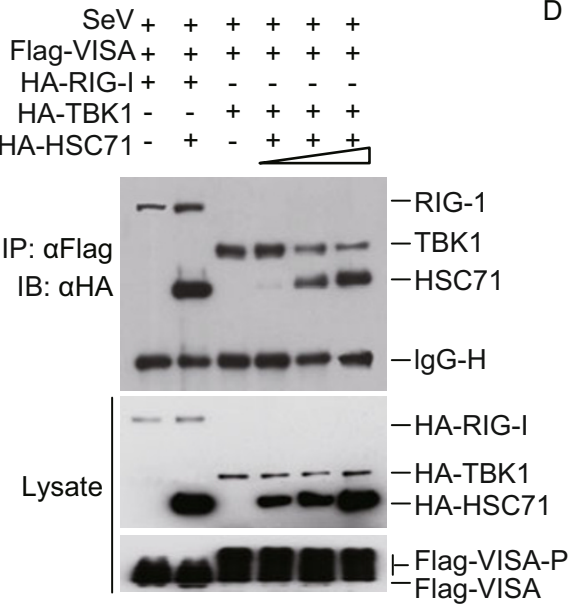

Flag-RIG-I - + + - +
Flag-VISA - - +-
HA-HSC71 + - - +

$\mathrm{HA}-\mathrm{HSC} 71+--+$
Myc-Ub +++++

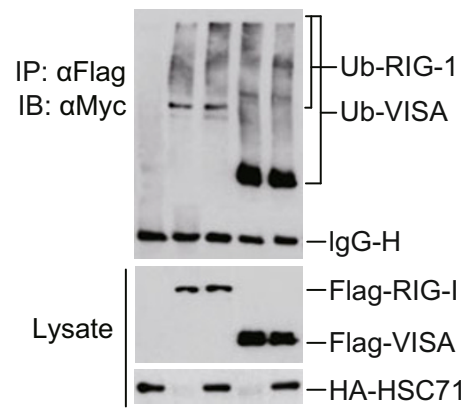

B

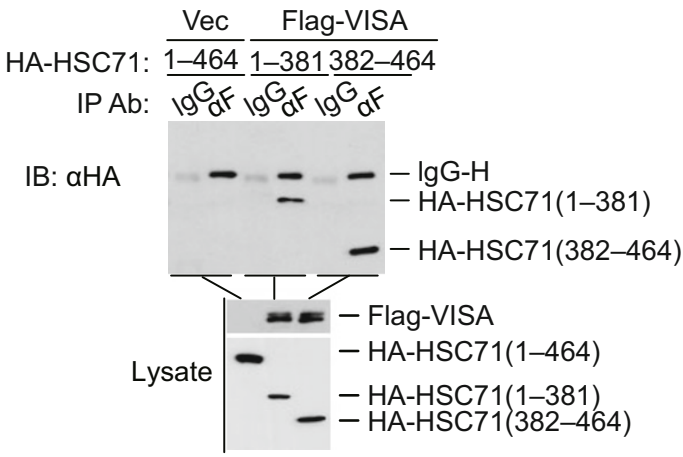

Flag-HSC71

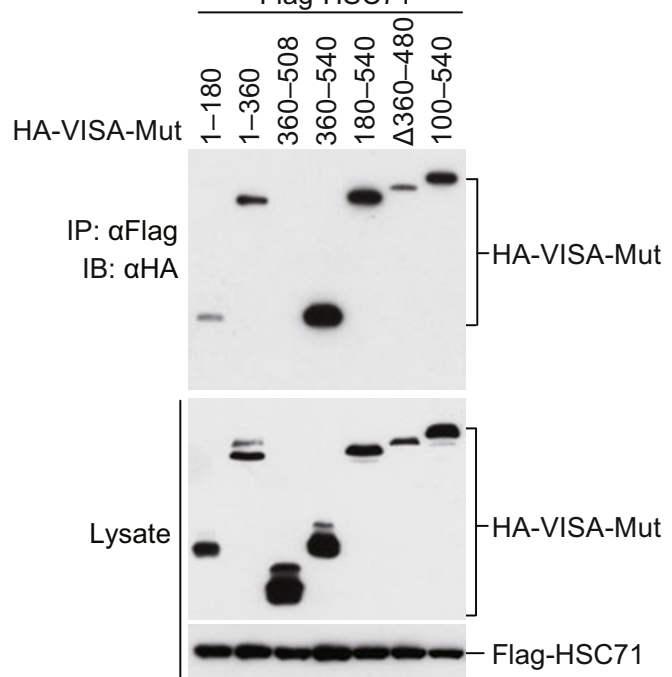

$\mathrm{E} \quad \mathrm{SeV}-\mathrm{C}_{-}+-++$

Flag-HSC71 - + - + HSC71-RNAi $-+-+$
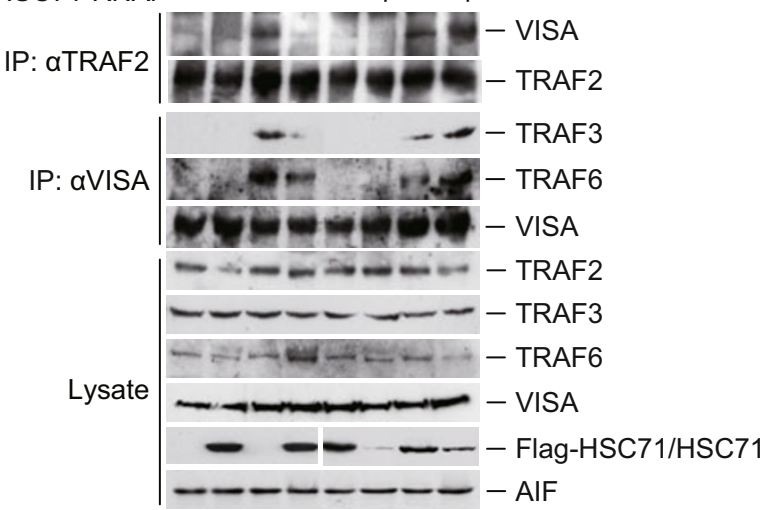

Figure 5. HSC71 impairs recruitment of downstream signaling components to VISA. (A) Endogenous HSC71 is associated with VISA. The 293 cells $\left(2 \times 10^{6}\right)$ were infected with $\mathrm{SeV}$ for $7 \mathrm{~h}$ or left uninfected. Immunoprecipitation and immunoblot analysis were performed with the indicated antibodies. (B) Domain mapping of the VISA-HSC71 interaction. The 293 cells $\left(1 \times 10^{6}\right)$ were transfected with the indicated plasmids $(5 \mu \mathrm{g}$ each). Cell lysates were immunoprecipitated with anti-Flag or control lgG. The immunoprecipitates were analyzed by immunoblotting with antiHA (top panel). The expression of the transfected proteins were analyzed by immunoblots with anti- Flag and anti- HA (middle and bottom panels) respectively. (C) HSC71 disrupts the association of VISA with TBK1 in a dose-dependent manner The 293 cells $\left(2 \times 10^{6}\right)$ were transfected with the indicated plasmids $(5 \mu \mathrm{g}$ each) and increase amounts of HSC71 expression plasmid. Afer transfection, the cells were infected with SeV for $6 \mathrm{~h}$. Coimmunoprecipitation and immunoblot analysis were performed as in (B). (D) HSC71 does not affect polyubiqutination and expression of VISA and RIG-I. The 293 cells $\left(1 \times 10^{6}\right)$ were transfected with wild-type ubiquitin expression plasmid $(1 \mu \mathrm{g})$ and the indicated plasmids $(5 \mu \mathrm{g}$ each). Coimmunoprecipitation and immunoblot analysis were performed as in (B). (E) HSC71 impairs the recruitment of TRAF2/3/6 to VISA. The 293 cells were transfected with the indicated overexpression plasmids. or RNAi plasmids. Twenty hours after transfection with the overexpression plasmids or $36 \mathrm{~h}$ after transfection with the RNAi plasmids, cells were left untreated or infected with $\mathrm{SeV}$ for $10 \mathrm{~h}$ before cell fractionation. The crude mitochondria were suspended in lysis buffer and treated by sonication before coimmunoprecipitation and immunoblot analysis with the indicated antibodies. 
A

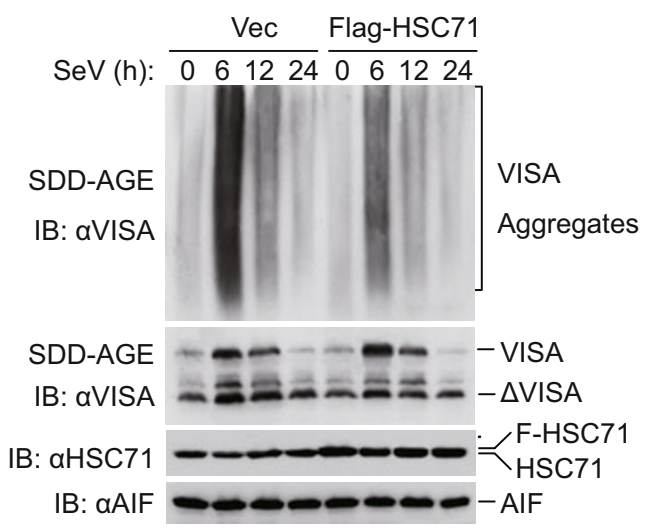

B

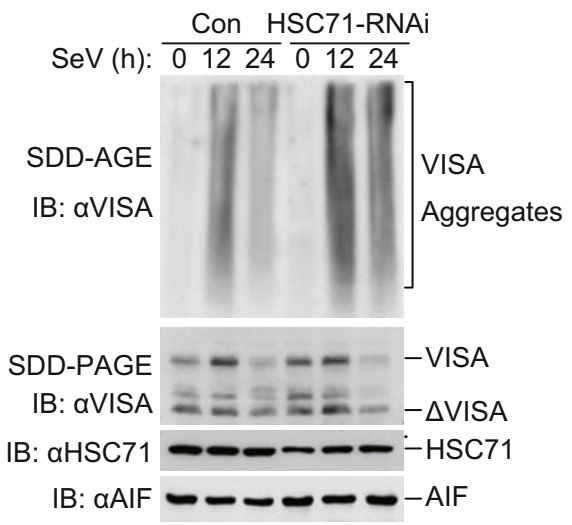

C

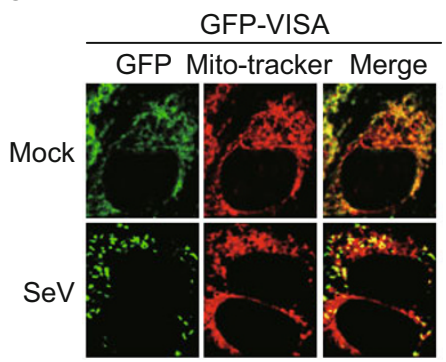

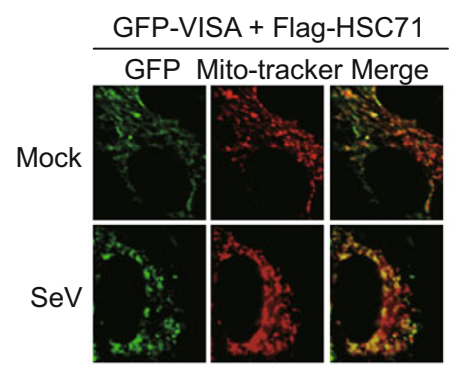

Figure 6. HSC71 regulates the formation of VISA aggregates. (A) Overexpression of HSC71 inhibits SeV-triggered formation of VISA aggregates. Crude mitochondrial extracts were prepared from 293 cells tranfected with HSC71 expression plasmid and infected with SeV for the indicated times. The extracts were fractionated by SDD-AGE and SDS-PAGE and analyzed by immunoblots with the indicated antibodies. (B) Knockdown of HSC71 potentiates SeV-triggered formation of VISA aggregates. Experiments were performed similarly as in (A) except that cells were transfected with HSC71-RNAi or GFP-RNAi plasmid before SeV infection. (C) HSC71 inhibits SeV-triggered formation of VISA aggregates in vivo. HeLa cells $\left(5 \times 10^{4}\right)$ were tranfected with GFP-VISA and Flag-HSC71. Twelve hours later, the cells were left untreated or infected with SeV for $8 \mathrm{~h}$. The cells were stained with Mito-Tracker Red for 30 min and then fixed with $4 \%$ paraformaldehyde for another $10 \mathrm{~min}$ before subjected for confocal microscopy.

\section{DISCUSSION}

Type I IFNs are critical mediators of innate antiviral responses. The production of type I IFNs is delicately regulated in a spatial-temporal manner by distinct molecules and mechanisms to avoid excessive immune responses. In this study, we identified HSC71 as an important regulatory component in the virus-triggered IFN- $\beta$ induction pathways. Overexpression of HSC71 inhibited SeV-triggered activation of IRF3, NF-KB and the IFN- $\beta$ promoter, whereas knockdown of HSC71 had an opposite effect. Consistently, overexpression of HSC71 reversed cytoplasmic poly $(\mathrm{I}: \mathrm{C})$ induced inhibition of VSV replication, whereas knockdown of HSC71 inhibited VSV replication. These results suggest that $\mathrm{HSC} 71$ negatively regulates virus-triggered IFN- $\beta$ induction and cellular antiviral response.

Several lines of evidences suggest that HSC71 inhibits virus-triggered signaling by targeting VISA. Firstly, overexpression of HSC71 inhibited VISA-, TBK1- but not TRIF- and IRF3mediated activation of ISRE, whereas knockdown of HSC71 potentiated VISA- but not TBK1- and IRF3-mediated activation of ISRE. In these experiments, inhibition of TBK1-mediated
ISRE activation by HSC71 was probably an aritifical effect because HSC71 did not inhibit ISRE activation mediated by TRIF, an upstream adapter protein of TBK1. Second, HSC71 interacted with VISA but not TBK1 both in vitro and in mammalian cells. Confocal microscopy demonstrated that HSC71 colocalized with VISA at the mitochondria. Third, overexpression of HSC71 impaired virus-induced recruitment of downstream signaling components to VISA, whereas knockdown of HSC71 enhanced the recruitment.

Recently, it has been demonstrated that in response to virus infection, the CARD domains of RIG-I is modified by K63-linked polyubiqutin chains, which then bind to the CARD domain of VISA and induces the formation of prion-like aggregates of VISA (Zeng et al., 2010; Hou et al, 2011). The VISA aggregates are formed fastly and resistant to protease digestion (Hou et al., 2011). These findings suggest that VISA is a robust booster in cellular antiviral response, and negative regulation targeting VISA aggregation is important to control excessive immune response. So far, how VISA aggregates are resolved in cells remains unknown. In this study, we found that overexpression of HSC71 inhibited the formation of VISA 
aggregates following viral infection, whereas knockdown of HSC71 promoted the formation of VISA aggregates after viral infection. Based on our data, we propose that HSC71 functions to prevent VISA aggregation under physiological conditions and dissolve VISA aggregates at the later period of virus infection to avoid an excess immune response. Since HSC71 is constitutively associated with VISA and its expression levels are not markedly changed following viral infection, the activity of HSC71 itself must be regulated by viral infection. Currently, the mechanisms on this regulation are unknown. Nevertheless, our findings reveal an important regulatory mechanism on virus-triggered IFN induction and cellular antiviral response.

\section{MATERIALS AND METHODS}

\section{Reagents}

Rabbit polyclonal antibodies against Myc tag (Cell Signaling Technology), AIF, IRF3, TRAF3, TRAF6; mouse monoclonal antibodies against TRAF2 (Santa Cruz Biotechnology), Flag, HA and $\beta$-actin (Sigma); horseradish peroxidase (HRP)-conjugated anti-mouse IgG and antirabbit IgG (Thermo Fisher and eBioscience); FuGENE6 transfection reagent (Roche Applied Science) and poly(I:C) (Invitrogen) were purchased from the indicated companies. Mouse or rabbit antisera against HSC71 were raised against recombinant human HSC71 protein. Sendai virus (SeV), VSV and rabbit anti-VISA antiserum were previously described (Zhong et al., 2008; Li et al., 2009; Lei et al., 2010; Mao et al., 2010; Wang et al., 2010; Li et al., 2011, 2012).

\section{Plasmid constructs}

NF-KB, ISRE, the IRF1 and IFN- $\beta$ promoter luciferase reporter plasmids, mammalian expression plasmids for Flag-, HA- or GFP-tagged VISA and its mutants, TBK1, TRIF, IRF3 and IRF3-5D (a constitutively active mutant of IRF3) have been previously described (Zhong et al., 2008; Li et al., 2009; Lei et al., 2010; Mao et al., 2010; Wang et al., 2010, 2011; Li et al., 2011; Chen et al., 2012; Li et al., 2012). CMV promoter-based mammalian expression plasmids for Flag-, HA- or CFP-tagged HSC71 were constructed by standard molecular biology techniques.

\section{Transfection and reporter assays}

The 293 cells $\left(1 \times 10^{5}\right)$ were seeded on 12-well plates and transfected $16 \mathrm{hr}$ later by standard calcium phosphate precipitation. In these experiments, empty control plasmid was added to ensure that the same amount of total DNA was transfected to each well. To normalize for transfection efficiency, $50 \mathrm{ng}$ of $\mathrm{pRL}-\mathrm{TK}$ Renilla luciferase reporter plasmid was added to each transfection. Approximately $18 \mathrm{~h}$ after transfection, luciferase assays were performed using a dual-specific luciferase assay kit (Promega). Firefly luciferase activities were normalized based on Renilla luciferase activities. All reporter assays were repeated for at least three times.

\section{Real-time PCR}

Total RNA was isolated from 293 cells using TRIzol reagent (Invitrogen) and subjected to real-time PCR analysis to measure expression of mRNAs. Gene-specific primer sequences were as follows: IFNB1:
TTGTTGAGAACCTCCTGGCT (forward), TGACTATGGTCCAGGCACAG (reverse); RANTES: GGCAGCCCTCGCTGTCATCC (forward), GCAGCAGGGTGTGGTGTCCG (reverse); TNFa: GCCGCATCGCCGTCTCCTAC (forward), СCTCAGCCCCСTCTGGGGTC (reverse); GAPDH: GAGTCAACGGATTTGGTCGT (forward), GACAAGCTTCCCGTTCTCAG (reverse).

\section{Immunoprecipitation and immunoblot analysis}

For transient transfection and coimmunoprecipitation experiments, 293 cells $\left(1 \times 10^{6}\right)$ were transfected with tested expression plasmids for $24 \mathrm{~h}$. The transfected cells were lysed in $1 \mathrm{~mL}$ of lysis buffer $(20 \mathrm{mmol} / \mathrm{L}$ Tris$\mathrm{HCl}, 150 \mathrm{mmol} / \mathrm{L} \mathrm{NaCl}, 1 \%$ Nonidet P-40, 1 mmol/L EDTA, $10 \mu \mathrm{g} / \mathrm{mL}$ aprotinin, $10 \mu \mathrm{g} / \mathrm{mL}$ leupeptin, $1 \mathrm{mmol} / \mathrm{L}$ phenylmethylsulfonylfluoride, $\mathrm{pH}$ 7.5). For each immunoprecipitation, $0.8-\mathrm{mL}$ aliquot of lysate was incubated with $0.5 \mu \mathrm{g}$ of the indicated antibody and $30 \mu \mathrm{L}$ of a $1: 1$ slurry of GammaBind G Plus-Sepharose (Amersham Biosciences) for $4 \mathrm{~h}$. The Sepharose beads were washed three times with $1 \mathrm{~mL}$ of lysis buffer containing $500 \mathrm{mmol} / \mathrm{L} \mathrm{NaCl}$. The precipitates were analyzed by standard immunoblotting procedures. For endogenous immunoprecipitation experiments, cells were infected with $\mathrm{SeV}$ for the indicated times or left uninfected. Cells were lysed in $5 \mathrm{~mL}$ of lysis buffer, and the lysate was incubated with $1 \mu \mathrm{L}$ of the indicated antiserum or preimmune serum. The subsequent procedures were carried out as described above.

\section{VSV plaque assays}

The 293 cells $\left(1 \times 10^{5}\right)$ were transfected with the indicated plasmids ( $0.5 \mu \mathrm{g}$ each). Eighteen hours later, cells were further transfected with poly(l:C) $(1 \mu \mathrm{g})$. Twenty-four hours after transfection, cells were infected with VSV [multiplicity of infection (moi), 0.1]. At $1 \mathrm{~h}$ after infection, cells were washed with PBS for three times and then medium was added. The supernatants were harvested at $24 \mathrm{hr}$ after washing. The supernatants were diluted $1: 10^{6}$ and then used to infect confluent BHK21 cells cultured on 24-well plates. At $1 \mathrm{~h}$ postinfection, the supernatant was removed, and $3 \%$ methylcellulose was overlayed. At 3 days postinfection, overlay was removed and cells were fixed with $4 \%$ formaldehyde for $20 \mathrm{~min}$, and then stained with $0.2 \%$ crystal violetin. Plaques were counted, averaged, and multiplied by dilution factors to determine viral titers as PFU/mL.

\section{RNA interference (RNAi)}

Double-strand oligonucleotides corresponding to the target sequences were cloned into the pSuper.Retro RNAi plasmid (Oligoengine). The following sequences were targeted for human HSC71 CDNA. \#1: TGACAAAGATGAAGGAAAT; \#2: GATGAAGGAAATTGCAGAA; \#3: GAACAAGAGAGCTGTAAGA. A pSuper.retro RNAi plasmid targeting GFP was used as control for all RNAi-related experiments.

\section{In vitro transcription/translation assays}

The tested proteins were expressed with a TNT Quick Coupled Transcription/Translation Systems kit (Promega) following instructions of the manufacturer.

\section{Subcellular fractionation}

Cellular fractionation experiments were performed as previously described (Zhong et al., 2008). In brief, 293 cells $\left(3 \times 10^{7}\right)$ infected with 
SeV or left uninfected for various time points were washed with PBS and lysed by douncing for 40 times in $3 \mathrm{~mL}$ of homogenization buffer (10 mmol/L Tris- $\mathrm{HCl}, \mathrm{pH} 7.4,2 \mathrm{mmol} / \mathrm{L} \mathrm{MgCl}_{2}, 10 \mathrm{mmol} / \mathrm{L} \mathrm{KCl}$, $250 \mathrm{mmol} / \mathrm{L}$ sucrose, $10 \mu \mathrm{g} / \mathrm{mL}$ aprotinin, $10 \mu \mathrm{g} / \mathrm{mL}$ leupeptin, $1 \mathrm{mmol} / \mathrm{L}$ phenylmethylsulfonylfluoride). The homogenate was twice centrifuged at $500 \mathrm{~g}$ for $10 \mathrm{~min}$. The supernatant was centrifuged at $5000 \mathrm{~g}$ for $10 \mathrm{~min}$ to precipitate crude mitochondria.

\section{Semidenaturing detergent agarose gel electrophoresis} (SDD-AGE)

SDD-AGE was performed as previously described (Hou et al., 2011). In brief, crude mitochondria were resuspended in 1x sample buffer (0.5× TBE, $10 \%$ glycerol, $2 \%$ SDS, and $0.0025 \%$ bromophenol blue) and loaded onto a vertical $1.5 \%$ agarose gel (Bio-Rad). After electrophoresis in the running buffer ( $1 \times$ TBE and $0.1 \%$ SDS) for 40 min with a constant voltage of $100 \mathrm{~V}$ at $4^{\circ} \mathrm{C}$, the proteins were transferred to Immobilon membrane (Millipore) for immunoblot analysis.

\section{Immunofluorescent confocal microscopy}

HeLa cells $\left(5 \times 10^{4}\right)$ were seeded on 24-well plates and transfected with the indicated palsmids by FuGENE transfection reagent. The transfected cells were incubated with the MitoTracker Red (Molecular Probes) for $30 \mathrm{~min}$. The cells were then fixed with $4 \%$ paraformaldehyde for $10 \mathrm{~min}$ and observed with an Olympus confocal microscope under a $100 \times$ oil objective.

\section{ACKNOWLEDGMENTS}

This work was supported by grants from the National Natural Science Foundation of China (Grant No. 30921001, 91029302, 31000639 and 31170792), and the National Basic Research Program (973 Program) (No. 2012CB910201).

\section{ABBREVIATIONS}

HSC, heat shock cognate; HSP, heat shock protein; IFN, interferon; ISRE, interferon-stimulated response element; MOI, multiplicity of infection; PAMP, pathogen-associated molecular pattern; PRR, pattern recognition receptor; RLR, RIG-I-like receptor; RNAi, RNA interference; SeV, Sendai virus; TLR, Toll-like receptor; TM, transmembrane; VSV, vesicular stomatitis virus

\section{COMPLIANCE WITH ETHICS GUIDELINES}

Zhigang Liu, Shu-Wen Wu, Cao-Qi Lei, Qian Zhou, Shu Li, Hong-Bing Shu, And Yan-Yi Wang declare that they have no conflict of interest.

This article does not contain any studies with human or animal subjects performed by the any of the authors.

\section{REFERENCES}

Akira, S., Uematsu, S., and Takeuchi, O. (2006). Pathogen recognition and innate immunity. Cell 124, 783-801.

Allen, I.C., Moore, C.B., Schneider, M., Lei, Y., Davis, B.K., Scull, M.A., Gris, D., Roney, K.E., Zimmermann, A.G., Bowzard, J.B., et al. (2011). NLRX1 protein attenuates inflammatory responses to infection by interfering with the RIG-I-MAVS and TRAF6-NF-kappaB signaling pathways. Immunity 34, 854-865.
Andrejeva, J., Childs, K.S., Young, D.F., Carlos, T.S., Stock, N., Goodbourn, S., and Randall, R.E. (2004). The V proteins of paramyxoviruses bind the IFN-inducible RNA helicase, mda-5, and inhibit its activation of the IFN-beta promoter. Proc Natl Acad Sci U S A 101, 17264-17269.

Chen, R., Li, M., Zhang, Y., Zhou, Q., and Shu, H.B. The E3 ubiquitin ligase MARCH8 negatively regulates IL-1beta-induced NF-kappaB activation by targeting the IL1RAP coreceptor for ubiquitination and degradation. Proc Natl Acad Sci U S A 109, 14128-14133.

Chen, T., and Cao, X. (2010). Stress for maintaining memory: HSP70 as a mobile messenger for innate and adaptive immunity. Eur $\mathrm{J} \mathrm{Im-}$ munol 40, 1541-1544.

Hou, F., Sun, L., Zheng, H., Skaug, B., Jiang, Q.X., and Chen, Z.J. (2011). MAVS forms functional prion-like aggregates to activate and propagate antiviral innate immune response. Cell 146, 448-461.

Jia, Y., Song, T., Wei, C., Ni, C., Zheng, Z., Xu, Q., Ma, H., Li, L., Zhang, Y., He, X., et al. (2009). Negative regulation of MAVSmediated innate immune response by PSMA7. J Immunol 183, $4241-4248$

Jounai, N., Takeshita, F., Kobiyama, K., Sawano, A., Miyawaki, A., Xin, K.Q., Ishii, K.J., Kawai, T., Akira, S., Suzuki, K., et al. (2007). The Atg5 Atg12 conjugate associates with innate antiviral immune responses. Proc Natl Acad Sci U S A 104, 14050-14055.

Kawai, T., Takahashi, K., Sato, S., Coban, C., Kumar, H., Kato, H., Ishii, K.J., Takeuchi, O., and Akira, S. (2005). IPS-1, an adaptor triggering RIG-I- and Mda5-mediated type I interferon induction. Nat Immunol 6, 981-988.

Lei, C.Q., Zhong, B., Zhang, Y., Zhang, J., Wang, S., and Shu, H.B. (2010). Glycogen synthase kinase 3beta regulates IRF3 transcription factor-mediated antiviral response via activation of the kinase TBK1. Immunity 33, 878-889.

Li, Q., Yan, J., Mao, A.P., Li, C., Ran, Y., Shu, H.B., and Wang, Y.Y. (2011). Tripartite motif 8 (TRIM8) modulates TNFalpha- and IL-1beta-triggered NF-kappaB activation by targeting TAK1 for K63-linked polyubiquitination. Proc Natl Acad Sci U S A 108, 19341-19346.

Li, Y., Chen, R., Zhou, Q., Xu, Z., Li, C., Wang, S., Mao, A., Zhang, X., He, W., and Shu, H.B. (2012). LSm14A is a processing bodyassociated sensor of viral nucleic acids that initiates cellular antiviral response in the early phase of viral infection. Proc Natl Acad Sci U SA 109, 11770-11775.

Li, Y., Li, C., Xue, P., Zhong, B., Mao, A.P., Ran, Y., Chen, H., Wang, Y.Y., Yang, F., and Shu, H.B. (2009). ISG56 is a negative-feedback regulator of virus-triggered signaling and cellular antiviral response. Proc Natl Acad Sci U S A 106, 7945-7950.

Li, Z., Menoret, A., and Srivastava, P. (2002). Roles of heat-shock proteins in antigen presentation and cross-presentation. Curr Opin Immunol 14, 45-51.

Liu, X.Y., Chen, W., Wei, B., Shan, Y.F., and Wang, C. (2011). IFNinduced TPR protein IFIT3 potentiates antiviral signaling by bridging MAVS and TBK1. J Immunol 187, 2559-2568.

Mao, A.P., Li, S., Zhong, B., Li, Y., Yan, J., Li, Q., Teng, C., and Shu, H.B. (2010). Virus-triggered ubiquitination of TRAF3/6 by clAP1/2 is essential for induction of interferon-beta (IFN-beta) and cellular antiviral response. J Biol Chem 285, 9470-9476.

Meylan, E., Curran, J., Hofmann, K., Moradpour, D., Binder, M., Bartenschlager, R., and Tschopp, J. (2005). Cardif is an adaptor protein in the RIG-I antiviral pathway and is targeted by hepatitis $\mathrm{C}$ 
virus. Nature 437, 1167-1172.

Moore, C.B., Bergstralh, D.T., Duncan, J.A., Lei, Y., Morrison, T.E., Zimmermann, A.G., Accavitti-Loper, M.A., Madden, V.J., Sun, L., Ye, Z., et al. (2008). NLRX1 is a regulator of mitochondrial antiviral immunity. Nature 451, 573-577.

Nakhaei, P., Mesplede, T., Solis, M., Sun, Q., Zhao, T., Yang, L., Chuang, T.H., Ware, C.F., Lin, R., and Hiscott, J. (2009). The E3 ubiquitin ligase Triad3A negatively regulates the RIG-I/MAVS signaling pathway by targeting TRAF3 for degradation. PLoS Pathog 5 , e1000650.

Seth, R.B., Sun, L., Ea, C.K., and Chen, Z.J. (2005). Identification and characterization of MAVS, a mitochondrial antiviral signaling protein that activates NF-kappaB and IRF 3. Cell 122, 669-682.

Srivastava, P.K., Menoret, A., Basu, S., Binder, R.J., and McQuade, K.L. (1998). Heat shock proteins come of age: primitive functions acquire new roles in an adaptive world. Immunity 8, 657-665.

Tang, E.D., and Wang, C.Y. (2010). TRAF5 is a downstream target of MAVS in antiviral innate immune signaling. PLoS One 5, e9172.

Triantafilou, M., and Triantafilou, K. (2004). Heat-shock protein 70 and heat-shock protein 90 associate with Toll-like receptor 4 in response to bacterial lipopolysaccharide. Biochem Soc Trans 32, 636-639.

Tsan, M.F., and Gao, B. (2004). Heat shock protein and innate immunity. Cell Mol Immunol 1, 274-279.

Wang, L., Liu, Y.T., Hao, R., Chen, L., Chang, Z., Wang, H.R., Wang, Z.X., and Wu, J.W. (2011). Molecular mechanism of the negative regulation of Smad1/5 protein by carboxyl terminus of Hsc70interacting protein (CHIP). J Biol Chem 286, 15883-15894.

Wang, Y.Y., Liu, L.J., Zhong, B., Liu, T.T., Li, Y., Yang, Y., Ran, Y., Li, S., Tien, P., and Shu, H.B. (2010). WDR5 is essential for assembly of the VISA-associated signaling complex and virus-triggered IRF3 and NF-kappaB activation. Proc Natl Acad Sci U S A 107, 815-820.
Wang, Y.Y., Ran, Y., and Shu, H.B. (2012). Linear ubiquitination of NEMO brakes the antiviral response. Cell Host Microbe 12, 129131.

Xu, L., Xiao, N., Liu, F., Ren, H., and Gu, J. (2009). Inhibition of RIG-I and MDA5-dependent antiviral response by $\mathrm{gC} 1 \mathrm{qR}$ at mitochondria. Proc Natl Acad Sci U S A 106, 1530-1535.

Xu, L.G., Wang, Y.Y., Han, K.J., Li, L.Y., Zhai, Z., and Shu, H.B. (2005). VISA is an adapter protein required for virus-triggered IFN-beta signaling. Mol Cell 19, 727-740.

Yasukawa, K., Oshiumi, H., Takeda, M., Ishihara, N., Yanagi, Y., Seya, T., Kawabata, S., and Koshiba, T. (2009). Mitofusin 2 inhibits mitochondrial antiviral signaling. Sci Signal 2, ra47.

Yoneyama, M., Kikuchi, M., Natsukawa, T., Shinobu, N., Imaizumi, T., Miyagishi, M., Taira, K., Akira, S., and Fujita, T. (2004). The RNA helicase RIG-I has an essential function in double-stranded RNAinduced innate antiviral responses. Nat Immunol 5, 730-737.

You, F., Sun, H., Zhou, X., Sun, W., Liang, S., Zhai, Z., and Jiang, Z. (2009). PCBP2 mediates degradation of the adaptor MAVS via the HECT ubiquitin ligase AIP4. Nat Immunol 10, 1300-1308.

Zeng, W., Sun, L., Jiang, X., Chen, X., Hou, F., Adhikari, A., Xu, M., and Chen, Z.J. (2010). Reconstitution of the RIG-I pathway reveals a signaling role of unanchored polyubiquitin chains in innate immunity. Cell 141, 315-330.

Zhong, B., Yang, Y., Li, S., Wang, Y.Y., Li, Y., Diao, F., Lei, C., He, X., Zhang, L., Tien, P., et al. (2008). The adaptor protein MITA links virus-sensing receptors to IRF3 transcription factor activation. Immunity 29, 538-550.

Zhong, B., Zhang, L., Lei, C., Li, Y., Mao, A.P., Yang, Y., Wang, Y.Y., Zhang, X.L., and Shu, H.B. (2009). The ubiquitin ligase RNF5 regulates antiviral responses by mediating degradation of the adaptor protein MITA. Immunity 30, 397-407. 